\title{
A CONSERVATIVE TREATMENT OF CHRONIC MIDDLE EAR SUPPURATION.*
}

By ANDREW CAMPBELL, M.B., F.R.C.S. (Edin.), Honorary Ear, Nose, and Throat Surgeon to the North Riding Infirmary and North Ormesby Hospital, Middlesbrough.

THE conservative treatment of chronic middle ear suppuration, associated with the name of Bezold, is widely known on the Continent, where it is a routine practice in many clinics. Bezold was the first to use boric acid in the treatment of suppurating ears in 1879, shortly after Lord Lister had established its antiseptic properties. A full account of the treatment is given in the Text-book of Otology by Bezold and Siebenmann. The necessary apparatus consists of the following: (I) A rubber bag (valveless Politzer bag) connected by about three feet of tubing to (2) Intratympanic cannulæ-four or five shapes and sizes. These were first introduced by Arthur Hartmann. (3) Probes wrapped with cotton wool. Wooden probes are excellent. (4) Two powder blowers, one for boric acid and the other for a mixture of salicylic acid and boric acid in the proportion of $I$ to 4. The salicylic acid is used in cases where there is cholesteatoma. (5) A Politzer bag.

The technique consists of five stages: (I) Intratympanic syringing with 4 per cent. boric lotion at body temperature. To obtain this temperature the bag is filled with lotion at $105^{\circ} \mathrm{F}$., and, if used immediately, the lotion is at the correct temperature. An intratympanic cannula of suitable size and shape is attached to the tubing and introduced through the perforation into the middle ear, care being taken that all air has been expelled from the system. The noisy escape of air into the middle ear is most alarming to the patient, who may make some sudden movement with unlooked-for results. The bag is now compressed gradually by a nurse, and the accessible parts of the middle ear are washed out in the view of the operator. It is most important that the lotion should be at body temperature, as otherwise nystagmus and giddiness result. (2) The meatus and parts of the middle ear accessible

* Reports for the year 1919 from the Ear and Throat Department of the Royal Infirmary, Edinburgh, under the care of A. Logan Turner, M.D., F.R.C.S.E. 


\section{Andrew Campbell}

through the perforation are dried by means of a probe and cotton wool. (3) The middle ear is politizerised so as to blow out any moisture which has escaped the drying. (4) A second and final drying, which must be very thorough. Malleable probes are of great value. (5) Powder all accessible parts of the middle ear and the outer surface of the tympanic membrane with a thin layer of boric acid. Where the perforation is small, the tympanum is not powdered. The powder may be blown through a dry intratympanic cannula in cases where the powder does not reach all parts. A small piece of cotton wool is inserted into the meatus. The treatment is repeated once daily; later, once every two days, and so on at longer intervals until the middle ear is dry as shown by the dry powder on examination. The treatment takes on an average from five to eight minutes for each patient.

Professor Nager of Zürich tells me that no serious complication has occurred in his clinic, where it is the only treatment adopted, nor has he heard of any fatalities. In nervous patients there is some difficulty at first, but it is surprising how quickly even very young children become tolerant to all intratympanic manipulations. This treatment should only be carried out by those who are accustomed to work in the ear, but there is no doubt that it is excellent training for ear work.

Bezold divides chronic suppurative otitis media into two great groups: (I) those with central perforations and (2) those with marginal perforations, in which are included perforations of Shrapnell's membrane, and total perforations. From the point of view of treatment the former are easy to cure and the latter more difficult, owing to the disease being more severe, less accessible, and often associated with cholesteatoma. The epithelium of the meatus easily grows through a marginal perforation to line bare areas in the middle ear in an attempt to bring about a natural cure. The successive shedding of this epithelium results in cholesteatoma.

\section{Results of Treatment of C.O.M.S. With Central PERFORATIONS.}

Von Ruppert reports 93 per cent. cures, of which 75 per cent. were cured within one month, and some of them, with discharge for years, were cured after one syringing (Nager). By cure is meant cessation of discharge only for short or long periods. 


\section{Chronic Middle Ear Suppuration}

In the series of cases detailed in this paper cure means something more definite. Bezold states: "Every circumscribed growth, be it ever so small, ought to be removed by means of a snare. I have never found a necessity for other operative interferences in chronic suppuration of the middle ear with central perforation, except in those extremely rare cases of general sepsis after acute recurrences." He reports, therefore, cure in all cases with central perforation by conservative means. This view is also held by Professor Nager. It must be mentioned here that anterior inferior central perforations are looked upon by them with much suspicion of being tubercular in origin. The claim as regards cure does not include tubercular cases.

\section{Results of Treatment of C.O.M.S. with Marginal PERFORATIONS.}

Von Ruppert reports 90 per cent. cures. Professor Nager claims about 70 per cent. of cures, including cases with cholesteatoma. He thinks that the radical mastoid operation is not justified in these cases, until this treatment has failed "The first sign of improvement is shown by less foetor, and shortly afterwards the discharge becomes less." Siebenmann is of opinion that if no improvement occurs in four to six weeks an operation should be done. Bezold writes: "Sometimes the suppuration remains foetid in spite of careful treatment, continued for months, by means of the antrum tube and the removal of the polypus. . . . This state of affairs proves that the entrance to the cavity is inadequate, and that a part of it cannot be reached by the jet of the syringe. The indication is, therefore, to make the focus of the disease accessible by means of an operation." He recommends first, the removal of the malleus and if this fails a radical mastoid operation, except in those cases where the ossicles are apparently intact and there is good hearing when an attempt is made to save the ossicles (modified radical mastoid). Certain Continental otologists, therefore, hold that all chronic suppurations with central perforations can be cured by conservative means, and that an operation is not justifiable except when there is an acute exacerbation. They also hold that a very large proportion of chronic suppurations with marginal perforations can be cured, even where there is cholesteatoma, and only cases which resist Bezold's treatment should be operated on. 


\begin{tabular}{|c|c|}
\hline & 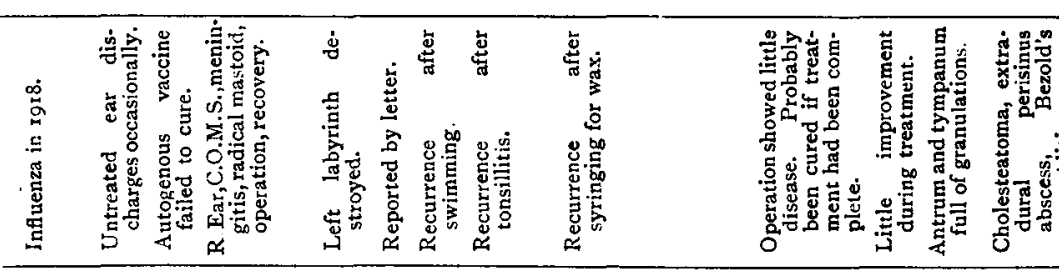 \\
\hline & 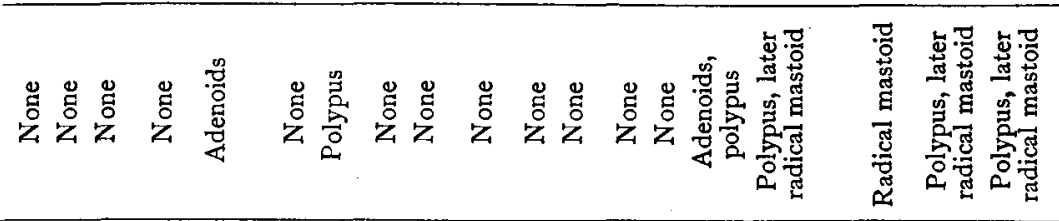 \\
\hline & 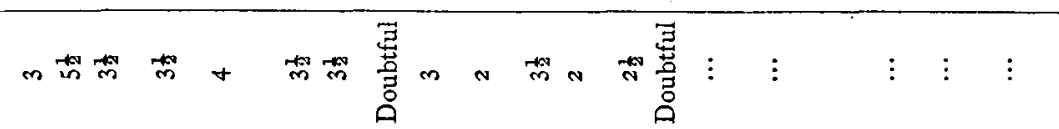 \\
\hline & 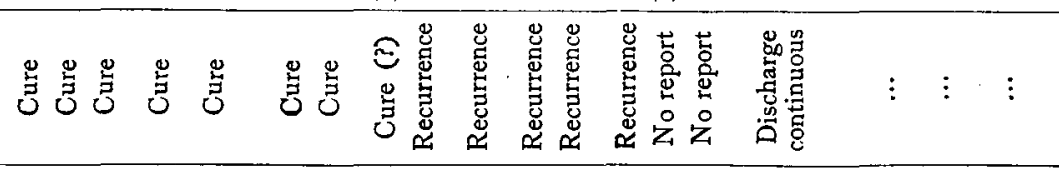 \\
\hline & 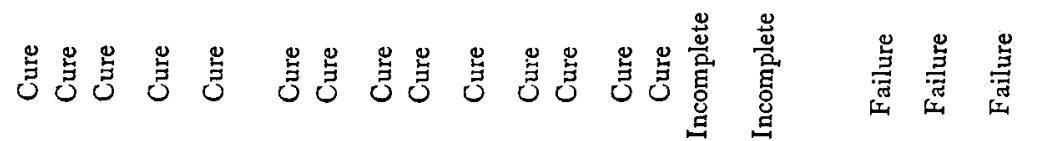 \\
\hline & 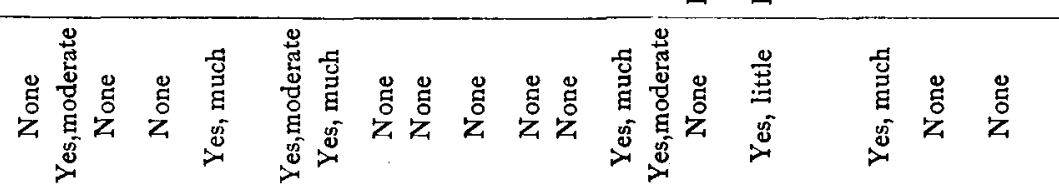 \\
\hline & 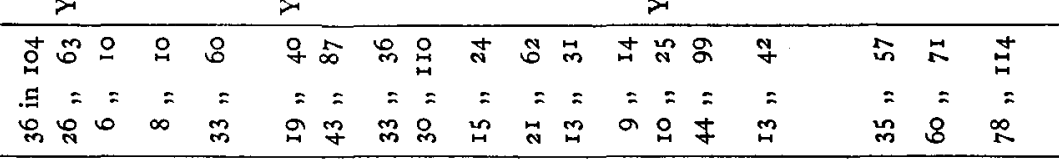 \\
\hline & 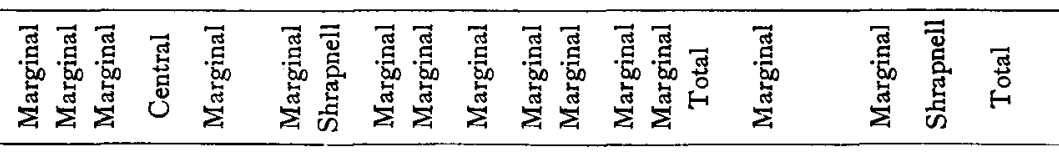 \\
\hline & 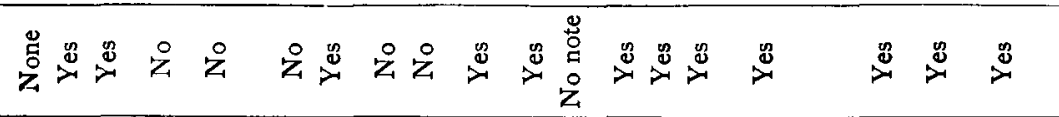 \\
\hline 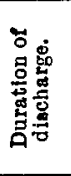 & 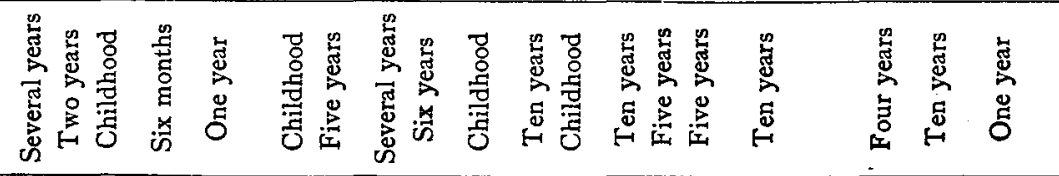 \\
\hline 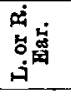 & 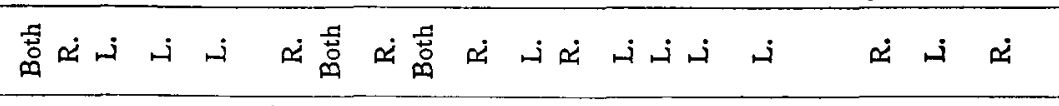 \\
\hline & 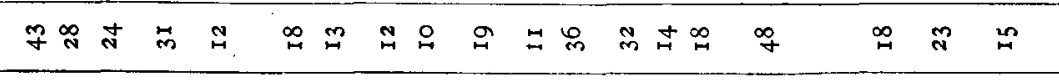 \\
\hline & نि: \\
\hline
\end{tabular}




\section{Chronic Middle Ear Suppuration}

Early in 1914, Dr Logan Turner and Dr J. S. Fraser suggested that I should treat some of these cases in Edinburgh and demonstrate the results. Only chronic cases, which resisted other methods, or cases which would as a matter of routine be entered for radical mastoid operation were selected. Nineteen cases were treated, of which seven were completely cured; that is to say, there was no history of recurrence, nor any evidence of suppuration on the date of the last examination in October I919-three to five years after treatment. Seven cases were. free of discharge for two or three years after treatment, and on recurrence the treatment was not available. In two cases the treatment was not completed, but in both, the ears were almost dry when the treatment was suspended. One of these has not reported since, and the other was operated on (see No. 16). Four cases eventually came to operation, including the one incompletely treated. The findings at the operations are of interest, especially in the one incompletely treated case, which showed a sclerotic mastoid, deep antrum with no pus, no cholesteatoma; long process of incus gone, malleus healthy, polypus, - a case which most probably could have been cured if treatment had been completed. If we consider that the arrest of discharge by treatment is a cure, then out of seventeen completed cases fourteen were cured. If treatment had been available on recurrence, no doubt some of the cases would still be free of discharge. One case recurred after tonsillitis, one after syringing for wax, and another after swimming. Most of the cases showed marked early improvement, say within fourteen days. In the fourteen cured cases the average number of treatments was twenty-two, and the average time of treatment forty-one days. Polypus and cholesteatoma are not contraindications for conservative treatment, though the cases with polypus were longer in being cured owing to the granulations left after removal. Cauterising with chromic acid beads did not hasten healing. Removal by the curette seems preferable. The size of some of the masses of cholesteatoma washed out was surprising, apparently much larger than the perforation.

Great care was taken as regards the temperature of the lotion used in syringing. There was no mishap during the whole investigation. All manipulations were done under the control of the eye. This necessarily means that the organisms coming away with the lotion during the syringing may infect the surgeon unless a mask is worn. Bezold warns those under- 


\section{Andrew Campbell}

taking this work of that possibility, and mentions that one or two of his assistants developed tuberculosis, possibly from this source.

Cases with central perforations may heal up quite well with syringing, drops, and drying thoroughly; a treatment which can be carried out by the patient and his family. When we come to persistent suppuration with marginal perforation, the result of this investigation may influence surgeons to try the Bezold treatment before advising the radical mastoid operation, which often involves a long and tedious after-treatment. The hearing also is seldom improved, especially when an epithelial graft is used, whereas in the conservative treatment a cure can be obtained in a large proportion of cases, with some improvement of hearing. It is noteworthy that in the three failures, which came to operation, the hearing distance was reduced in each. It would be interesting to try this treatment with a view to hastening recovery, in cases where a modified radical operation has been done.

My thanks are due to Dr Logan Turner for permission to treat his patients and to publish these results. The work was carried out in the Outpatient Clinic of the Ear and Throat Department in the Royal Infirmary, Edinburgh. The operations were performed by Dr J. S. Fraser, whose remarks have been quoted in two cases. I thank Dr Fraser for his interest in this treatment and for his advice on many occasions. I am much indebted to Professor Nager of Zuirich, who pointed out the excellent results obtained by the Bezold School, and taught me the technique of this treatment.

References.-Bezold, "Zur Antiseptischen Behandlung der Mittelohreiterungen," Arch. f. Ohrenhlk., vol. xv. 188o, Lehrbuch der Ohrenheilkunde, Wiesbaden, I906 (translated into English by J. Hollinger, M.D., of Chicago). Nager (Zürich), "Uber die Heutigen Anschauungen der Konservativen Behandlung Chronischer Mittelohreiterungen," Medizinischen Klinik, No. 38, 1909. V. Ruppert, "Zur Behandlung und Prognose der Chronischen Mittelohreiterung," Mïnch. Med. Wochschr., 1908 No. 2 I. 